\title{
Boosted Gabor Features Applied to Vehicle Detection
}

\author{
Hong Cheng, Nanning Zheng, Chong Sun \\ Institute of Artificial Intelligence and Robotics, \\ Xi' an Jiaotong University, China. \\ hcheng@mail.xjtu.edu.cn,nnzheng@mail.xtju.edu.cn \\ Huub van de Wetering \\ Technische Universiteit Eindhoven, The Netherlands
}

\begin{abstract}
Robust vehicle detection is a challenging task given vehicles with different types, and sizes, and at different distances. This paper proposes a Boosted Gabor Features $(B G F)$ approach for vehicle detection. The two main conventional Gabor filter design approaches are a filter bank design approach with fixed parameters even for different applications and a learning approach. In contrast, the parameters of our boosted Gabor filters, learned from examples, differ from application to application. Moreover, our boosted approach optimizes the filter parameters for every image sub-window, and the boosted filters have a large response for sub-windows containing a part of a vehicle resulting in a greatly improved performance in vehicle detection.

Our vehicle detection has two basic phases in which we build a multi-resolution hypothesis-validation structure. In the vehicle hypothesis generation phase, hypothesis lists are generated for three ROIs with different resolutions using horizontal and vertical edges, and following that, a hypothesis list for the whole image is obtained by combining these three lists. In the subsequent hypothesis validation phase, we validate the vehicle hypothesis list by inputting the boosted Gabor feature vector into the support vector machine.

In the context of vehicle detection, the resulting system yields detection rates comparable to the best previous systems while achieving a 20 frames per second real-time performance on a Pentium(R)4 CPU $2.4 \mathrm{GHz}$.
\end{abstract}

\section{Introduction}

According to the statistics of China's Ministry of Public Safety, traffic accidents cause on average one injury every minute and one death every 5 minutes in China. Clearly,
Intelligent Driver-Assistance and Safety Warning (IDASW) Systems can be of use. In this paper we look in particular at vehicle detection and tracking for such a system.

Currently, IDASW systems based on Radar have a higher cost than those based on machine vision, while having narrow field of view and bad lateral resolution. In Adaptive Cruise Control (ACC) systems, a camera can detect the cutin and overtaking vehicle from the adjacent lane earlier than the radar. Due to these reasons, it is more difficult to apply such radar-based systems into practical IDASW systems. Consequently, robust and real time vehicle detection in video attracts more attention of scholars all over the world [2] [3] [8].

\section{Previous Work}

D. Gabor first proposed 1D Gabor function in 1946 and J.G. Daugman extended it to 2D later. In fact, a Gabor filter is a local bandpass filter that can reach the theoretical limit for the spatial domain and the frequency domain simultaneously. Consequently, Gabor filters have been successfully applied into object representation in various computer vision applications, such as texture segmentation and recognition [6], face recognition [10], scene recognition, and vehicle detection [8].

The basic issue of a Gabor filter is how to select the parameters of a filter that responds mainly to an interesting object, such as a vehicle or a pedestrian. Accurate detection only occurs if the parameters defining Gabor filters are well selected. Three main approaches have been proposed in the literature for selecting Gabor filters for object representation: manual selection, Gabor filter bank design (including filter design) [6], and a learning approach [8] [9] [10] [7]. In [1], Ilkka Autio proposed an approach for manual selection: An initial set of Gabor filters were experimentally selected from a larger set and then manually tuned. In general, a Gabor filter bank design defines a small filter pool, 
and determines the parameters of its filters independent of the application domain; moreover, the bandwidth of those Gabor filter design approaches can not be determined autonomously. In image browsing and retrieval, a strategy is used to ensure that the half-peak magnitude support of the filter responses in the frequency domain touch each other by using a filter bank with 6 directions and 4 scales to compute the features of a texture [5]. Due to independence between filter bank and the application domain, such an approach can be used for object classification, detection and tracking. The main problems of this filter design approach are small filter pool sizes, no prior knowledge, and poor performance. Learning-based Gabor filter design approaches select the Gabor filters according to its application domain. Du-Ming Tsai proposed an optimization algorithm for Gabor filters using a simulated annealing approach to obtain the best Gabor filter in texture segmentation [9]. A face recognition application using a strong classifier cascaded by weak classifiers was proposed by S.Z. Li; in his approach weak classifiers were constructed based on both magnitude and phase features from Gabor filters [10].

In terms of vehicle detection, Alberto Broggi introduced a multi-resolution vehicle detection approach, and proposed to divide the image into three fixed ROIs[2]. His approach allows detection of multiple vehicles in a predefined region. However, it uses a symmetry axis for detecting vehicles that is not only time-consuming to compute but symmetry features are somewhat problematic. In [8], Zehang Sun proposed an Evolutionary Gabor Filter Optimization (EGFO) approach for vehicle detection, and used the statistical feature of the response of selected Gabor filters to classify the test image using a trained SVM classifier. Although good performance has been reported, EGFO has large computational cost for selection of a Gabor filter. Moreover, each Gabor filter is optimized for a complete image, but it is applied to each sub-window of a test image, which reduces the quality of representation.

To reduce the computational burden and improve the performance in vehicle detection, we propose a supervised learning approach based on boosted Gabor features. A similar attempt to select the Gabor features is described in [7]. However, the choosed Gabor feature set in that study is larger than those in our study; moveover, the SVM is only used to classify objects during the period of recognizing step rather than the previous training step. Their approach may result in performance decrease. In contrast, we use SVM as classifier during the period of both training step and classifying step.

\section{Boosted Gabor Features Using AdaBoost}

\subsection{Gabor Feature}

We first introduce some necessary definitions for Gabor filters and basic concepts for vehicle representation. The 2D Gabor function can be defined as follows.

$$
G_{p}(u, v)=\frac{1}{2 \pi \sigma_{u} \sigma_{v}} e^{-\frac{1}{2}\left(\frac{U^{2}}{\sigma_{u}^{2}}+\frac{V^{2}}{\sigma_{v}^{2}}\right)} \cdot e^{2 \pi j \hat{f} U}
$$

Where

$$
\left\{\begin{array}{l}
U=(u, v) \cdot(\cos \varphi, \sin \varphi) \\
V=(-u, v)(\sin \varphi, \cos \varphi)
\end{array}\right.
$$

and $\hat{f}$ is the radius frequency of a complex sinusoidal signal modulating Gaussian function, $\varphi$ is the direction of a Gabor filter, $\sigma_{u}$ and $\sigma_{v}$ are the scale parameters of the filter, and $p=\left(\hat{f}, \varphi, \sigma_{u}, \sigma_{v}\right) \in R^{4}$ represents all the parameters of a Gabor filter. Clearly, for image pixel set $\Omega$, Gabor features can be obtained by convolving the input image $\mathrm{I}(\mathrm{u}, \mathrm{v})((u, v) \in \Omega)$ and a $2 \mathrm{D}$ Gabor filter $g(u, v)$ written by

$$
R(u, v)=\iint_{\Omega} I(\xi, \eta) g(u-\xi, v-\eta) d \xi d \eta .
$$

Although a linear feature could be directly used to represent an object, few scholars do that. The most used Gabor features are thresholded Gabor features, Gabor-energy features, Complex moment Gabor features, and grating cell operator features. In this paper, we adopt the complex moment features of a Gabor filter response as the feature vector of our classifier.

\subsection{Boosted Gabor Features}

Selection of different Gabor features has some effect on detection performance, however, the primary issue of selecting the Gabor filter is to find the Gabor filters strongly responding to the object of interest. The filter parameters are adjusted to obtain the strongest response for subwindows comprising a vehicle part. The image is divided into 9 overlapping sub-windows, and the vehicle is represented with statistical features, mean $\mu$, standard deviation $\theta$, and the skewness $\kappa$, from a convolution between a subwindow and a Gabor filter [8].

Having obtained Gabor features of an object, it is time to evaluate the performance of a Gabor feature. Boosting approaches aim at improving the accuracy of any given learning algorithm and focusing on "difficult" examples. AdaBoost algorithm proposed by E. Schapire is one of the most popular variations of basic boosting algorithms [4]. In its original form, it is used to improve the accuracy of any given learning algorithm. In this paper, it is used to boost the Gabor features for vehicle detection. 
There are many Gabor features associated with a subwindow; however, few Gabor features are crucial for vehicle detection. Consequently, feature selection must be performed on these Gabor features. In this paper, we optimize the SVM classifier parameters for each of the nine sub-windows, and then test the resulting nine classifiers for each sub-window using test examples recording the classification rate of each Gabor filter for the 9 different subwindows. According to the results, we perform the boosting task on a larger set of Gabor features using AdaBoost algorithm, where each round of boosting finds one Gabor feature for a sub-window from the candidate features. After $\mathrm{T}$ iteration, it yields $\mathrm{T}$ Gabor filters for each sub-window (See Table 1). In our experiments, a total of 36 filters are combined into a feature vector to represent vehicles. The detection performance of BGF approach using the features after 4 iterations is better than those after 6 iterations.

The selection of a Gabor filter is to find the optimal parameter set in Gabor parameter space $R^{4}$

$$
\left\{p_{1}, \cdots, p_{i}, \cdots, p_{N}\right\}
$$

where $p_{i}=\left(\hat{f}_{i}, \varphi_{i}, \sigma_{u i}, \sigma_{v i}\right)$, and $N$ is the number of Gabor filters. For convenience of computing Gabor parameters are discretized. We define the range of $\hat{f}$ to be $\left[\hat{f}_{\min }, \hat{f}_{\max }\right]$. According to the Nyquist theorem, the digital frequency $\omega=\pi$ corresponds to the maximum frequency of a band limited signal, and the frequencies higher than $\pi$ will be distorted. We write $\omega_{\max }=2 \pi f_{\max } / f_{s}=2 \pi \hat{f}_{\max }$, and then $\hat{f}_{\text {max }}=\omega_{\max } / 2 \pi$, where $f$ is the general frequency, $f_{s}$ is the sampling frequency, and $\hat{f}_{k}$ is the $k$-th normalized frequency that can be discretized by

$$
\hat{f}_{k}=\hat{f}_{\text {min }}+\frac{\hat{f}_{\text {max }}-\hat{f}_{\text {min }}}{L} a^{k} \text { with } a=\left(\frac{\hat{f}_{\text {max }}}{\hat{f}_{\text {min }}}\right)^{\frac{1}{L-1}}
$$

where $L$ is the number of sample points and $a$ is the sample scale. For the direction $\varphi$ of a filter, the filter response to an object in $[0, \pi]$ is the same as to an object in $[\pi, 2 \pi]$.

The sample interval for uniform sampling is $\Delta \varphi=$ $180 / P$ degrees, where $P$ is the number of samples for $\varphi$. The scale parameters $\sigma_{x}$ and $\sigma_{y}$ are actually the effective size of a Gaussian function, and their ranges are equal, say $\left[\sigma_{\min }, \sigma_{\max }\right]$. The upper limit $\sigma_{\max }=W_{s} / 5$, where $W_{s}$ is the sub-window width, resulting in $98.7 \%$ energy in the range $[-\pi, \pi]$ of $\varphi$. At the same time, the lower limit $\sigma_{\min }$ equals 0 , and the number of samples for both $\sigma_{x}$ and $\sigma_{y}$ is $M$.

In our experiments in this paper, $a=1.5588, L=15$, $P=15, M=10, N=22500, W_{s}=40, \hat{f} \in$ $\left[\hat{f}_{\text {min }}, \hat{f}_{\text {max }}\right]=[0,0.5]$, and $\sigma_{x}, \sigma_{y} \in=[0,8]$. Fig. 1 shows our boosted Gabor filters for vehicle detection.

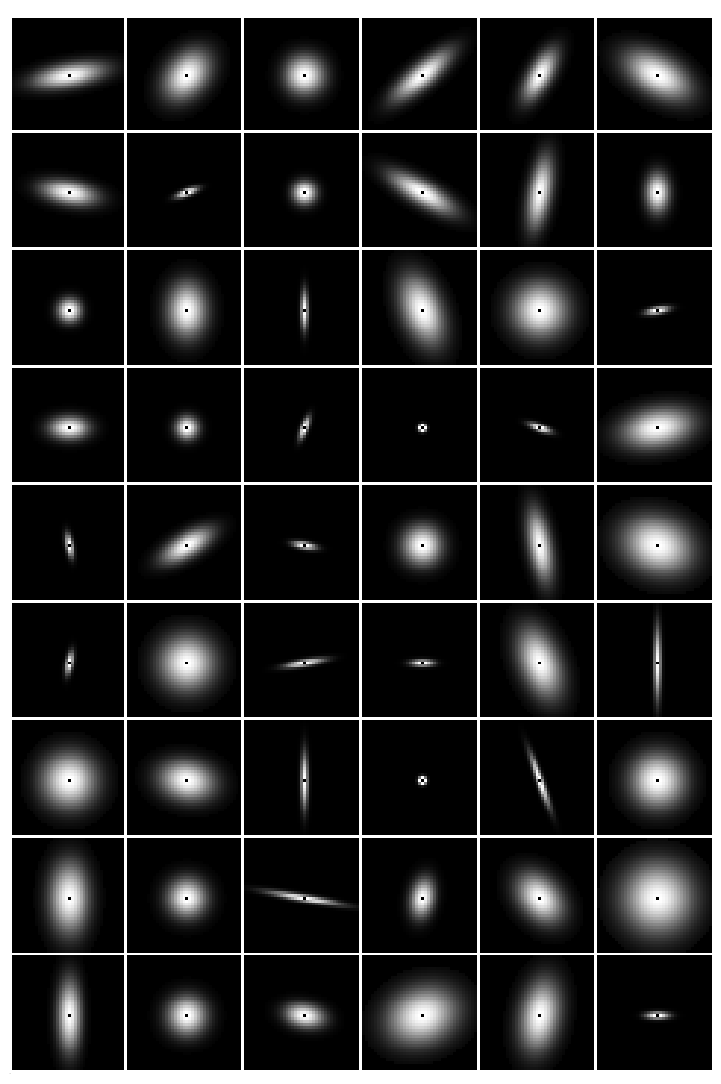

Figure 1. Boosted Gabor filters using AdaBoost algorithm: each row shows the boosted Gabor filters for one sub-window, and the $i$-th column represents the Gabor filter after the $i$-th iteration.

\section{Application to Vehicle Detection}

\subsection{SVM Classifier}

SVM is an efficient approach to find the optimal hyperplane in a binary classification: this hyperplane has the maximum margin between two distinguished classes, which ensures not only the minimum empirical risk, but also the minimum Vapnik Chervonenkis (VC) confidence. If the training examples from two classes cause the two classes' margin to be maximal, then the classification hyperplane satisfies the following equation:

$$
f(x)=\sum_{i=1}^{L} y_{i} a_{i} k\left(x, x_{i}\right)+b
$$

where $x, x_{i} \in \Re^{N}$ are $\mathrm{N}$-dimensional input feature vectors, $L$ is the number of examples, $y_{i} \in(-1,1)$ is the label of 


\section{Table 1. BGF Algorithm Description}

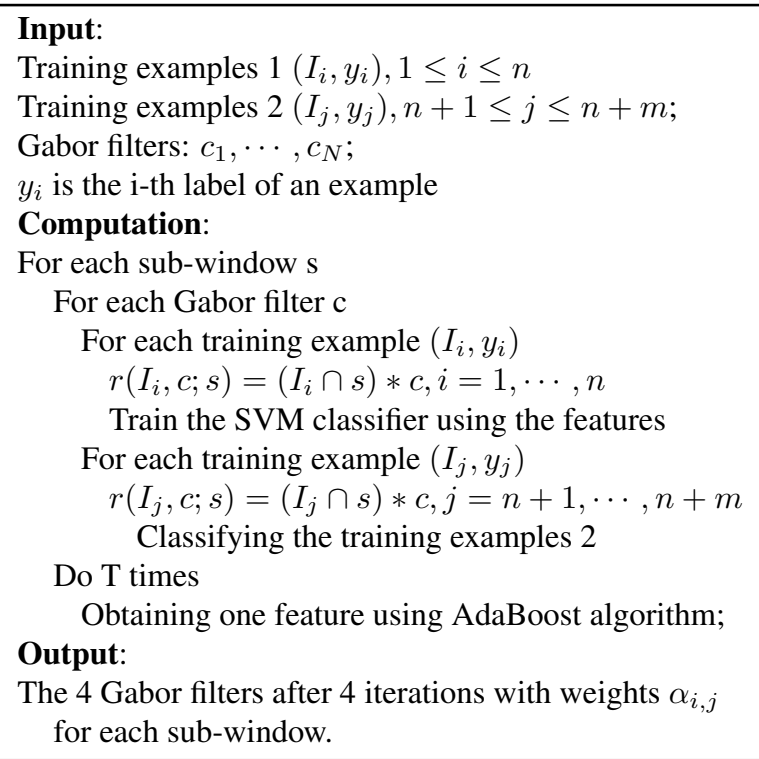

the $i$-th example, and $k\left(x, x_{i}\right)$ is the inner product function. We use radial basis function as kernel function, defined as

$$
k\left(x, x_{i}\right)=\exp \left(-\frac{\left\|x-x_{i}\right\|^{2}}{2 \sigma^{2}}\right)
$$

\subsection{Hypothesis-Validation Structure for Vehicle Detection}

Inspired by A. Broggio[2], we extract three ROIs, a near one, one in the middle, and a far one, from a $640 \times 480$ image. His approach uses fixed regions at the cost of flexibility, we remove this limitation and build a simple and efficient hypothesis-validation structure in 3 steps. (1) We generate ROI Candidates using a vanishing point of the road in the original image. (2) We create a multi-resolution vehicle hypothesis based on the preceding candidate regions. From the analysis of edge histograms, we generate hypotheses for each ROI and combine them into a single list. (3) We conduct vehicle validation using the boosted Gabor features and SVM classifier. According to the judging of the classifier, we determine whether hypotheses represent a vehicle or a non-vehicle (see [3], for details).

\section{Experimental Results and Analysis}

We have carrried out vehicle dectection using, apart from our approach, the EGFO approach and a no-boosting approach. The distribution of Gabor filter parameters , $(\hat{f}, \varphi$, $\left.\sigma_{x}, \sigma_{y}\right)$, are shown in Fig.1. We can see that our boosted
Gabor filters are different from the optimized Gabor filters using EGFO because each Gabor filter in our approach is optimized for a sub-window rather than for a complete image. For the frequency of Gabor filters, the boosted filters tend to have a low frequency due to large structures in vehicles, like windows and bumpers. are more The directions of most of the boosted Gabor filters are close to $0^{\circ}, 45^{\circ}$, $90^{\circ}, 135^{\circ}$ (see 2nd sub-figure of Fig.1), due to prevalence of these angles in vehicles. In addition, from the latter two sub-figures of Fig.1, it follows that $\sigma_{y}$ is larger than $\sigma_{x}$, in accordance with vehicles being wider than high. To summarize, the choice of Gabor filter parameters is heavily dependent on the detection object, a Gabor filter that works well for vehicles probably does not work for pedestrians, and vice versa. Our Gabor filter selection optimized for vehicle detection results in better performance than those based on previous selection methods. For training the classifier,
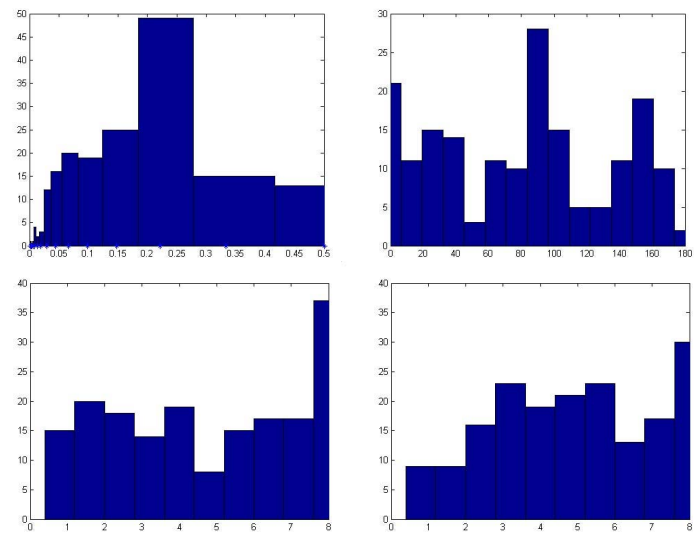

Figure 2. The Distribution of the Parameters of Gabor Filters

we selected 500 images from our vehicle database which was collected in Xi' an in 2005. They contain 1020 positive examples and 1020 negative examples. In testing the classifier, we use 500 negative and positive examples independent of the training examples.

For the validation of the performance of our BGF approach, the vehicle detection experiments were performed on three different optimization approaches for 22,500 Gabor filters. The experimental results show the Average Right Rate (ARR) of a no-boosting Gabor feature approach is $90 \%$, that of BGF approach is $96 \%$, and that of EGFO approach is $91 \%$. Fig.4(a) is the comparison of our detector with the other two approaches, and the Receiver Operating Characteristics (ROC) curves that compare different boosting approaches are shown in Fig.4(b). These figures show that our vehicle detector has a good discriminability while a low decision bias comparing both no-boosting and EGFO 


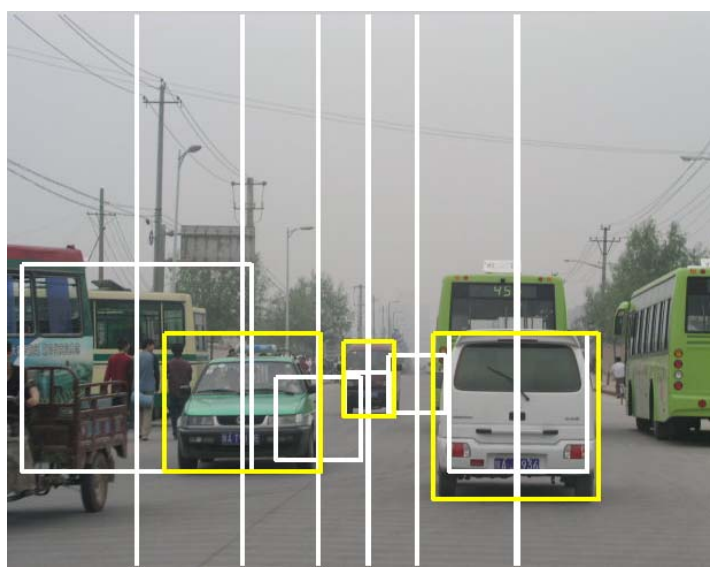

Figure 3. Vehicle Detection Based on Hypothesis-Validation

algorithms.

\section{Conclusions and Future Work}

In this paper we have introduced a boosting approach for Gabor filters, and a structure of hypothesis and validation for vehicle classification and detection. The experimental results of our system so far show that the algorithm has good classification and detection performance for on-road realtime vehicle detection. Further research work will focus on other detection tasks, such as pedestrian detection and face detection.

\section{References}

[1] I. Autio and T. Elomaa. Flexible view recognition for indoor navigation based on Gabor filters and support vector machines. Pattern Recognition, 36:2769-2779, 2003.

[2] A. Broggi, P. Cerri, and P. C. Antonello. Multi-resolution vehicle detection using artificial vision. IEEE Intelligent Vehicle Symposium, Parma, Italy, pages 14-17, June 2004.

[3] H. Cheng, N. N. Zheng, C. Sun, and H. van de Wetering. Vanishing point and Gabor feature based multi-resolution on-road vehicle detection. In International Symposium on Neural Networks, 2006.

[4] Y. Freund and R. E. Schapire. A decision-theoretic generalization of on-line learning and an applications to boosting. In Computational Learning Theory: Eurocolt'95, 1995.

[5] B. S. Manjunath and W. Y. Ma. Texture features for browsing and retrieval of image data. IEEE Transaction on Pattern Analysis Machine Intelligence, 18(8):837-842, 1996.

[6] T. P.Weldon, W. E.Higgins, and D. F.Dunn. Efficient Gabor filter design for texture segmentation. Pattern Recogniton, 29(12):2005-2015, 1996.

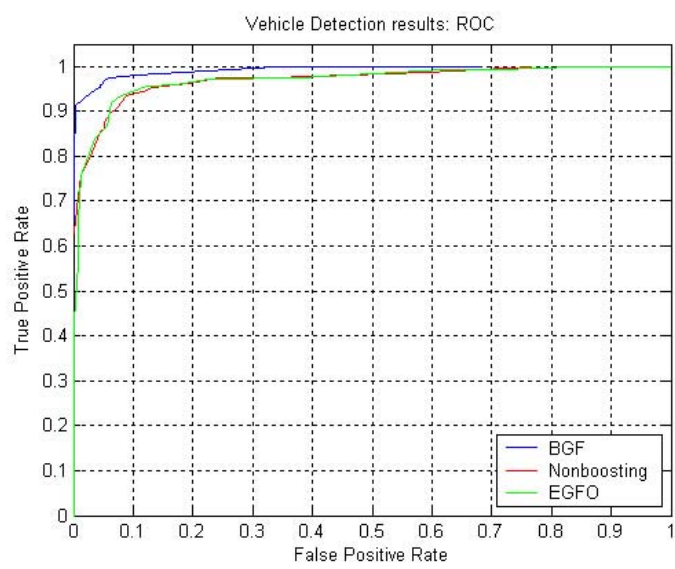

(a) ROC curves

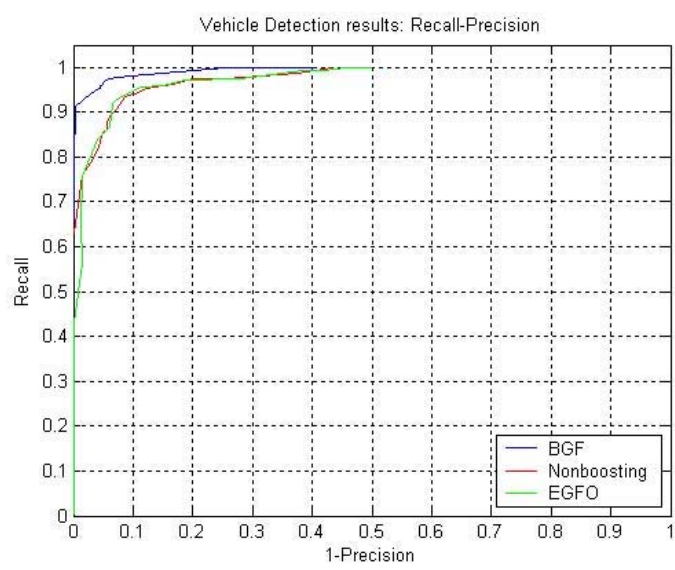

(b) Recall-precision curves

Figure 4. Two kinds of curves comparing different Gabor filter optimization approaches

[7] L. L. Shen and L. Bai. Adaboost gabor feature selection for classification. In Proceedings of Image and Vision Computing New Zealand, Akaroa, New Zealand, November 21-23, 2004.

[8] Z. Sun, G. Bebis, and R. Miller. On-road vehicle detection using evolutionary Gabor filter optimization. IEEE Transaction on Intelligent Transportation Systems, 6(4):125-137, 2005.

[9] D.-M. Tsai, S.-K. Wu, and M.-C. Chen. Optimal Gabor filter design for texture segmentation using stochastic optimization. Image and Vision Computing, 19(4):299-316, 2001.

[10] L. Zhang, S. Li, Z. Qu, and X. Huang. Boosting local feature based classifiers for face recognition. The first IEEE workshop on Face Processing in Video, pages 2005-2015, June 282004. 